ARTICLE

\title{
A sister lineage of the Mycobacterium tuberculosis complex discovered in the African Great Lakes region
}

\author{
Jean Claude Semuto Ngabonziza (10) 1,2,3,16, Chloé Loiseau4,5,16, Michael Marceau,16, Agathe Jouet (i) 7, \\ Fabrizio Menardo (1) 4,5, Oren Tzfadia², Rudy Antoine (10 6 , Esdras Belamo Niyigena', Wim Mulders (D) ${ }^{2}$, \\ Kristina Fissette ${ }^{2}$, Maren Diels ${ }^{8}$, Cyril Gaudin ${ }^{7}$, Stéphanie Duthoy7, Willy Ssengooba (i) ${ }^{9}$, Emmanuel André ${ }^{10}$, \\ Michel K. Kaswa ${ }^{11}$, Yves Mucyo Habimana (10 ${ }^{12}$, Daniela Brites (10 4,5, Dissou Affolabi ${ }^{13}$, Jean Baptiste Mazarati ${ }^{14}$, \\ Bouke Catherine de Jong (1) 2, Leen Rigouts (1) 2,3, Sebastien Gagneux (10) 4,5,17凶, \\ Conor Joseph Meehan (i) $2,15,17 \bowtie$ \& Philip Supply (iD) 6,17凶
}

The human- and animal-adapted lineages of the Mycobacterium tuberculosis complex (MTBC) are thought to have expanded from a common progenitor in Africa. However, the molecular events that accompanied this emergence remain largely unknown. Here, we describe two MTBC strains isolated from patients with multidrug resistant tuberculosis, representing an as-yet-unknown lineage, named Lineage 8 (L8), seemingly restricted to the African Great Lakes region. Using genome-based phylogenetic reconstruction, we show that L8 is a sister clade to the known MTBC lineages. Comparison with other complete mycobacterial genomes indicate that the divergence of $L 8$ preceded the loss of the cobF genome region - involved in the cobalamin/vitamin B12 synthesis - and gene interruptions in a subsequent common ancestor shared by all other known MTBC lineages. This discovery further supports an East African origin for the MTBC and provides additional molecular clues on the ancestral genome reduction associated with adaptation to a pathogenic lifestyle.

\footnotetext{
${ }^{1}$ National Reference Laboratory Division, Department of Biomedical Services, Rwanda Biomedical Center, Kigali, Rwanda. ${ }^{2}$ Mycobacteriology Unit, Department of Biomedical Sciences, Institute of Tropical Medicine, Antwerp, Belgium. ${ }^{3}$ Department of Biomedical Sciences, Antwerp University, Antwerp, Belgium. ${ }^{4}$ Swiss Tropical and Public Health Institute, Basel, Switzerland. ${ }^{5}$ University of Basel, Basel, Switzerland. ${ }^{6}$ Univ. Lille, CNRS, Inserm, CHU Lille, Institut Pasteur de Lille, U1019 - UMR 9017, CIIL - Center for Infection and Immunity of Lille, 59000 Lille, France. ${ }^{7}$ Genoscreen, Lille, France. ${ }^{8}$ BCCM/ITM, Mycobacterial Culture Collection, Institute of Tropical Medicine, Antwerp, Belgium. ${ }^{9}$ Department of Medical Microbiology, College of Health Sciences, Makerere University, Kampala, Uganda. ${ }^{10}$ Laboratory of Clinical Bacteriology and Mycology, Department of Microbiology and Immunology, KU LeuvenLeuven, Belgium. ${ }^{11}$ National Tuberculosis Program, Kinshasa, DR, Congo. ${ }^{12}$ Tuberculosis and Other Respiratory Diseases Division, Institute of HIV/AIDS Disease Prevention and Control, Rwanda Biomedical Center, Kigali, Rwanda. ${ }^{13}$ Laboratoire de Référence des Mycobactéries, Cotonou, Benin. ${ }^{14}$ Department of Biomedical Services, Rwanda Biomedical Center, Kigali, Rwanda. ${ }^{15}$ School of Chemistry and Biosciences, University of Bradford, Bradford, UK. ${ }^{16}$ These authors contributed equally: Jean Claude Semuto Ngabonziza, Chloé Loiseau, Michael Marceau. ${ }^{17}$ These authors jointly supervised this work: Sebastien Gagneux, Conor Joseph Meehan, Philip Supply. ${ }^{凶}$ email: sebastien.gagneux@swisstph.ch; c.meehan2@bradford.ac.uk; philip.supply@ibl.cnrs.fr
} 
T uberculosis (TB), caused by members of the Mycobacterium tuberculosis complex (MTBC), is among the ancient scourges of humankind ${ }^{1}$, and remains the leading cause of mortality globally due to an infectious disease ${ }^{2}$. Intense research has been dedicated to decipher the evolutionary history of the MTBC and to understand the causes underlying the worldwide spread of $\mathrm{TB}^{3-5}$. Current genome data show that the MTBC is comprised of the five human-adapted lineages representing $M$. tuberculosis sensu stricto (L1-4 and L7), two other humanadapted lineages traditionally referred to as M. africanum (L5-6) and at least nine animal-adapted lineages ${ }^{6}$. Africa is the only continent where all MTBC lineages are present, suggesting that the MTBC emerged from a common ancestor therein and then expanded to the rest of the world following human migrations $s^{3,7-10}$. However, the genomic traits of this common ancestor and the region from which this expansion took place in Africa remain unknown. Whole-genome sequencing (WGS) analyses showed that rare human TB bacilli with a smooth colony morphotype, highly restricted to the Horn of Africa and named Mycobacterium canettii (alias smooth tubercle bacilli or STB) represent early evolutionary branching lineages that predate the emergence of the most recent common ancestor (MRCA) of the MTBC (or of the rest of the MTBC, if M. canettii is considered to be part of the complex $)^{4,11,12}$. Indeed, whereas known MTBC strains differ by no more than $\sim 2000$ single nucleotide polymorphisms $(\mathrm{SNPs})^{13}$, M. canettii strains are 10- to 25 -fold more genetically diverse and separated by at least 14,000 SNPs from the hitherto known MTBC MRCA ${ }^{4,12}$. Moreover, M. canettii strains are less virulent and possess highly mosaic genomes, possibly reflecting adaptation to an environmental reservoir favouring active lateral gene flow ${ }^{4,14-16}$. These biological differences support the existence of lineages that reflect intermediate stages in the evolution from M. canettii towards the obligate MTBC pathogens.

Here, we describe two exceptional strains representing a new clade, diverging before the MRCA of the other MTBC lineages. These two strains were discovered in two independent analyses and were both multidrug-resistant (MDR; i.e. resistant to at least rifampicin and isoniazid). One was isolated from a TB patient in Rwanda through an ongoing MDR-TB diagnostic trial in Africa. The second isolate came from a patient in Uganda, and was discovered upon screening publicly available draft genome datasets, where it was misclassified as an M. bovis strain. We used PacBio and Illumina WGS to reconstruct the full circular genome of the Rwandan strain. We used these data and the available Illumina sequencing data of the Ugandan strain to reconstitute the phylogeny of this novel lineage, which we named Lineage 8 (L8), and further investigate molecular and evolutionary events associated with the emergence of the MTBC.

\section{Results}

Patient with the L8 MTBC strain in Rwanda. The Rwandan strain was isolated from a male patient, aged 77 years, HIVnegative, resident of Rulindo district bordering with the Southwest of Uganda, and who had lived in Uganda previously. The patient was diagnosed with rifampicin-resistant $\mathrm{TB}$ based on standard Xpert MTB/RIF testing (Xpert; Cepheid, Sunnyvale, CA, USA), which probes for mutations in the rifampicin resistancedetermining region of the $r p o B$ gene of the $\mathrm{MTBC}^{17}$. The results of the assay showed a rare delayed probe $B$ reaction ( $\sim 3 \%$ prevalence in Rwanda) ${ }^{18}$, presumed (and later confirmed; see below) to be due to the rifampicin resistance-associated Asp435Tyr mutation $^{19}$.

Per routine practice, the patient was initiated on standard shortcourse MDR-TB treatment (i.e. 9-month WHO-endorsed MDRTB regimen, including moxifloxacin, kanamycin, protionamide, ethambutol, clofazimine, high dose isoniazid and pyrazinamide $)^{20}$. However, the patient developed hypotension, and eventually died due to probable cardiac failure, after 20 days of treatment. Phenotypic drug-susceptibility testing (DST) confirmed resistance to both rifampicin and isoniazid, and susceptibility to other antiTB drugs including ethambutol, fluoroquinolones and second-line injectables.

Growth characteristics and biochemical properties of the Rwandan L8 strain. The Rwanda strain was grown in 12.5 days on Mycobacterial Growth Indicator Tubes. Colonies were observed on the fifth week after initial inoculation on Löwenstein-Jensen medium, indicating a slow grower phenotype with rough colonies (Fig. 1). The strain also displayed archetypal biochemical characteristics of $M$. tuberculosis sensu stricto, including niacin production combined with urease hydrolysis (Table 1).

Genotypic resistance and SNP profile of the Rwandan L8 strain by Deeplex-MycTB. Following the MDR-TB diagnosis, the strain was included in the first set of tests for an ongoing MDR-TB diagnostic trial 'DIAgnostics for MDR-TB in Africa (DIAMA) Clinicaltrials.gov, NCT03303963', evaluating a new targeted deep sequencing assay, called Deeplex-MycTB. Deeplex-MycTB testing confirmed the presence of the $r p o B$ Asp435Tyr mutation conferring rifampicin resistance, along with the inhA Ser94Ala mutation conferring isoniazid resistance, consistently with the MDR phenotype identified by phenotypic DST (Fig. 2). This strain also harboured two alleles in phylogenetic positions in embB (Ala378) and gidB (Ala205) not associated with resistance to ethambutol or streptomycin, which were both shared by several MTBC lineages (L1, 5, 6, 7 and animal lineages) and $M$. canettii ${ }^{21}$. In addition, eight other-so far uncharacterised non-synonymous SNPs were identified in six of the 18 gene targets interrogated by the assay (Fig. 2). Moreover, this test detected an atypical spoligotype pattern, 1111100000000000000000000000000001110000000 (Fig. 2), which was further confirmed by conventional membrane-based spoligotyping testing. This spoligotype pattern was unique in the global spoligotype database that comprises 111,637 MTBC isolates from 131 countries $^{22}$.

WGS analysis and phylogenetic position of the Rwandan and Ugandan L8 strains. Results from WGS analysis of the Rwandan strain using Illumina sequencing confirmed all Deeplex-MycTB findings.

The strain isolated in Uganda was discovered independently upon screening global MTBC genome datasets publicly available on NCBI/EBI, comprising $\sim 20,000$ genomes in total. From subsequent processing with our WGS analysis pipeline, we found 1 genome that did not classify in any of the 7 humanadapted lineages or 9 animal-adapted ecotypes known at the time, but had been misclassified as $M$. bovis isolated from a human patient ${ }^{23}$. These WGS data revealed a similar spoligotype 1111100000000000000000000000000001111000111 , with an overlapping signature characterised by the presence of spacers 1 to 5 and 34 to 37 (vs $34-36$ in the Rwandan strain) with all intervening spacers missing. Moreover, the Ugandan strain also shared the same $r p o B$ Asp435Tyr and inhA Ser94Ala mutations and the same sequence alleles in $e m b B$ and gidB. The Ugandan strain contained an additional katG Ser315Thr mutation conferring high-level isoniazid resistance, as well as the embA $\mathrm{C}-11 \mathrm{~A}$ and $e m b B$ Asp328Tyr mutations predictive of ethambutol resistance and two $p n c A$ missense mutations, predictive of pyrazinamide resistance. Moreover, only three of the nine aforementioned uncharacterised SNPs detected by DeeplexMycTB were shared between both strains. 


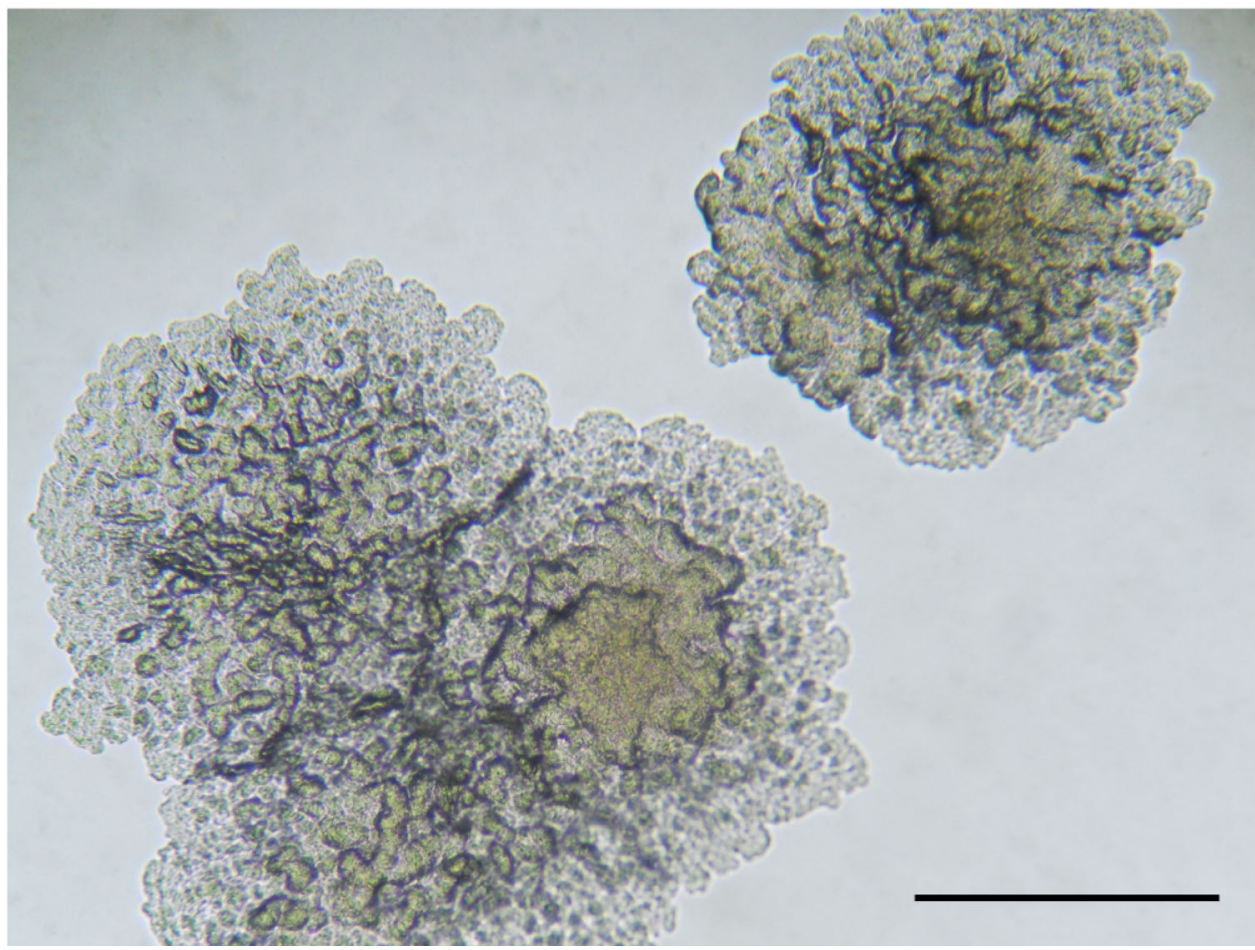

Fig. 1 Image of colonies from the Rwandan Lineage 8 strain. Isolate grown on solid medium, with the typical rough morphotype of Mycobacterium tuberculosis. Colonies grown on Dubos agar medium were visualized by microscopy. The scale bar represents $\sim 2 \mathrm{~mm}$.

Table 1 Standard biochemical characteristics of selected mycobacterial species and $\boldsymbol{M}$. tuberculosis complex lineages/ subspecies.

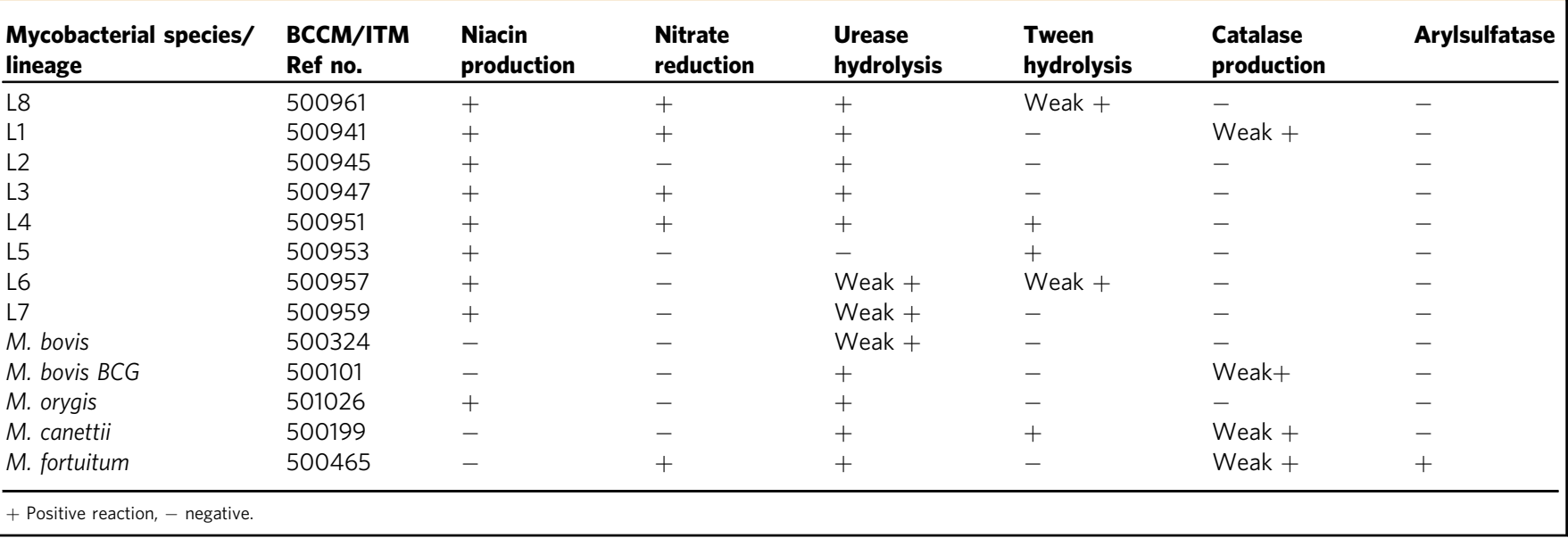

To further assess the relationships between both strains and in comparison to other MTBC strains, a maximum likelihood phylogeny was inferred from 241 MTBC genomes, including representatives of all known human- and animal-adapted lineages ${ }^{6}$ and using an $M$. canettii strain as an outgroup. $M$. canettii represents the closest outgroup to the MTBC including L8, as shown by subsequent comparative analysis of a complete L8 genome (see below), and previous observations of $\sim 2.0 \mathrm{Mb}$ larger genomes and substantially lower average nucleotide identities of phylogenetically closest non-tuberculous mycobacterial species such as $M$. marinum and $M$. kansasii ${ }^{4,24,25}$. This reconstruction revealed a unique phylogenetic position of the two new genomes from Rwanda and Uganda (Fig. 3), representing a newly characterised monophyletic clade, in which none of the known
MTBC genomes were contained. A core genome-based phylogeny was also constructed from representatives of the MTBC lineages as well as M. canettii, M. marinum and M. kansasii (Supplementary Fig. 1). This phylogeny confirmed the placement of the L8 Rwandan strain as being a new clade between $M$. canettii and the other lineages of the MTBC. Based on these phylogenies, this clade shares a MRCA with the rest of the MTBC, thus representing a new sister clade to the known MTBC, which we named Lineage 8 (L8). Comprehensive SNP analysis identified a total of 189 SNPs separating both L8 genomes, which is within the range of zero to 700 SNPs found between any two strains within any of the lineages 1 to 7 of the $\mathrm{MTBC}^{13}$. On average, two strains of the MTBC including L8 differed by 1443 SNPs (corresponding to $0.04 \%$ of the genome, excluding repetitive/'problematic' regions), thus at least an 


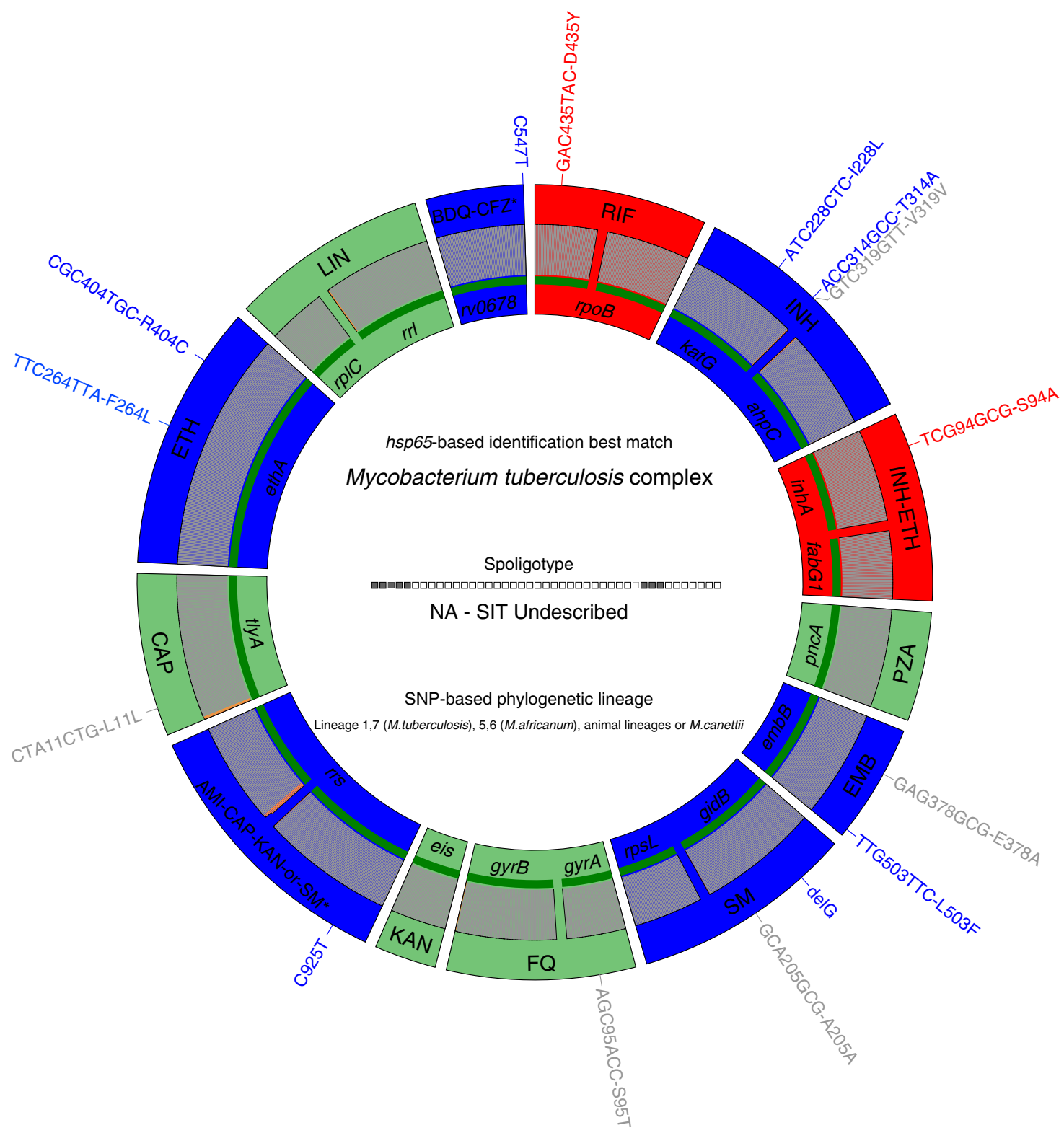

Fig. 2 Deeplex-MycTB results identifying a MDR-TB strain from Rwanda with an atypical genotypic profile. Deeplex-MycTB results identifying a MDRTB strain from Rwanda with an atypical genotypic profile. Target gene regions are grouped within sectors in a circular map according to the tuberculous drug resistance with which they are associated. The two sectors in red indicate regions where rifampicin and isoniazid resistance-associated mutations are detected. The multiple sectors in blue refer to regions, where as yet uncharacterised mutations are detected, whereas sectors in green indicate regions where no mutation or only mutations not associated with resistance (shown in grey around the map) were detected. Green lines above gene names represent the reference sequences with coverage breadth above $95 \%$. Limits of detection (LOD) of potential heteroresistance (reflected by subpopulations of reads bearing a mutation), depending on the coverage depths over target sequence positions, are represented by grey (LOD 3\%) and orange zones (variable LOD > 3-80\%; only seen in extremities of a few targets, such as the two rrs regions) above the reference sequences within the sectors.

Information on an unrecognised spoligotype, an equivocal SNP-based phylogenetic lineage and on mycobacterial species identification, based on hsp65 sequence best match, are shown in the centre of the circle. AMI amikacin, BDQ bedaquiline, CAP capreomycin, CFZ clofazimine, EMB ethambutol, ETH ethionamide, FQ fluoroquinolones, KAN kanamycin, LIN linezolid, INH isoniazid, PZA pyrazinamide, RIF rifampin, SM streptomycin, SIT spoligotype international type.

order of magnitude lower than the SNP distance separating any MTBC strain from M. canettii ${ }^{4,13}$.

The absence of any matching pattern in the global spoligotype database, as well as the lack of detection of this clade in previous large WGS datasets of MTBC strains from global sources, suggests that L8 is rare and seemingly geographically restricted to the African Great Lakes region. Specifically, the L8 spoligotype signature and the three SNPs shared by both L8 strains were not detected in any of 115 MTBC genomes from a previous drug resistance survey in Uganda ${ }^{26}$, nor in 380 rifampicin-resistant strains from Rwanda collected between 1991 and 2018, from routine drug resistance surveillance as well as various drug resistance surveys ${ }^{27-29}$. Furthermore, among 14 other available isolates out of 27 from Uganda and Rwanda tested by Gene Xpert $\mathrm{MTB} / \mathrm{RIF}$ that showed the same delayed probe $\mathrm{B}$ as L8, none displayed the L8 signatures when tested by Deeplex-MycTB or by classical spoligotyping. Likewise, none of $>1500$ clinical samples from TB patients tested by Deeplex-MycTB from a 


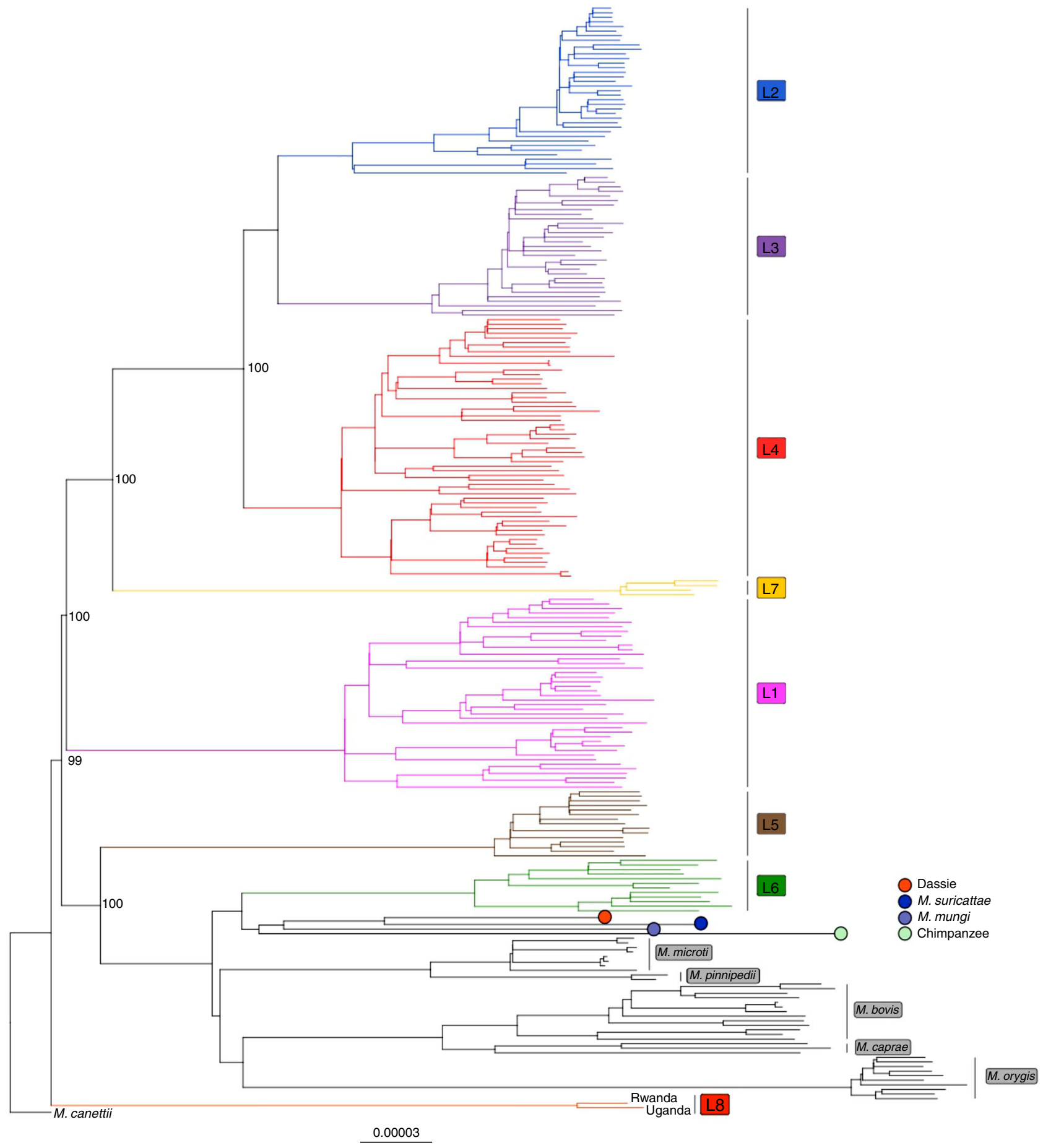

Fig. 3 Maximum likelihood phylogeny of 241 MTBC genomes, inferred from 43,442 variable positions. Maximum likelihood phylogeny of 241 MTBC genomes, inferred from 43,442 variable positions. The scale bar indicates the number of substitutions per polymorphic site. Branches corresponding to human-adapted strains are coloured and branches corresponding to animal-adapted strains are depicted in black. The phylogeny is rooted on $M$. canettii and bootstrap values are shown for the most important splits.

recent nationwide drug resistance survey performed in the Democratic Republic of the Congo displayed the L8 spoligotype signature or the specific SNPs.

Defining features of a complete $\mathbf{L 8}$ genome. To further assess the phylogenetic position of $\mathrm{L} 8$ and its split from the rest of the MTBC, the Rwandan strain was subjected to long read-based PacBio sequencing. Comparison of the obtained assembly with 36 available complete genomes of MTBC members, comprising L1L4 (including $\mathrm{H} 37 \mathrm{Rv}$ ), M. africanum (L6) and M. bovis strains, showed a highly syntenic organisation, with no major structural rearrangement between both groups. Although the assembled L8 genome of $4,379,493 \mathrm{bp}$ was within the $4.34-4.43 \mathrm{Mb}$ size range of the other MTBC genomes, it was $30 \mathrm{~kb}$ smaller than the $4.41-\mathrm{Mb}$ mean size of genomes of $M$. tuberculosis sensu stricto ${ }^{30}$. However, the largest part of this gap was accounted by the absence of three genomic regions in L8, corresponding to regions 
of difference (RDs) known to be variably present or absent in other MTBC (sub)lineages ${ }^{31,32}$ (Supplementary Data 1). These include a $9.3 \mathrm{~kb}$ PhiRv1 prophage region (RD3), as well as $10.0 \mathrm{~kb}$ and $8.5 \mathrm{~kb}$ segments corresponding to RD14 and RD5, comprising the $p l c D$ gene region and the $p l c A B C$ gene cluster, respectively ${ }^{31}$. In $\mathrm{L} 8$, each of the two latter regions only contained one copy of the IS6110 insertion sequence, devoid of direct repeats (DRs) that normally flank IS6110 copies after transposition, indicating that these deletions in L8 resulted from recombination between two adjacent IS6110 copies with loss of the intervening sequences ${ }^{33}$. These mobile DNA-related deletions, which also arose independently in several other MTBC branches ${ }^{31,34}$, probably occurred after the divergence of L8 from the other MTBC lineages. Apart from these three deletions and two dozen repetitive/multicopy genes (IS6110-related, PE/PPE-, or Mce-encoding), we only found 5 non-repetitive genes, included in two small segments $(3.4 \mathrm{~kb}$ and $4.4 \mathrm{~kb})$, which were undetected in the complete L8 genome while being present in reference MTBC genomes (Supplementary Data 1).

Conversely, a $4.4 \mathrm{~kb}$ genome region was present in the genomes of both L8 strains and in M. canettii, but absent in the 36 available complete genomes of MTBC members (Supplementary Data 2). This region comprises the $c o b F$ gene (Fig. 4), encoding the precorrin $6 \mathrm{~A}$ synthase involved in the cobalamin/vitamin B12 synthesis, along with two other genes, respectively encoding a PE-PGRS protein family member and a protein of unknown function. This region shared by the L8 and the M. canettii genomes is also present in the phylogenetically proximal non-tuberculous mycobacterial species M. marinum and M. kansasii (Fig. 4). Via BLAST analysis, we further confirmed the systematic absence of cobF in any of 6456 quality draft genome assemblies available as of January 2020 from the NCBI, from strains belonging to lineages $1-7$ or the animal lineages of the MTBC. Moreover, we thereby determined that the junction between the sequences flanking the cobF deletion was at the same nucleotide position in all but 6 of these genomes, resulting in the truncation of $r v 0943 c$ and $r v 0944$ genes as seen in the complete MTBC genomes (Fig. 4). Consistent with the clonal evolution of the MTBC with negligible, if any, horizontal gene transfer between strains $^{1,14,32,35}$, the perfect conservation of this sequence junction suggests that $c o b F$ was lost in the MRCA of the other MTBC lineages, after its divergence from L8. The 6 exceptions were 3 strains from lineage 4.3 and 3 strains from lineage 3 that showed slightly larger deletions, including the $5^{\prime}$ region of $r v 0943 c$ or the $5^{\prime}$ region of $r v 0943 c, r v 0944$ and the $5^{\prime}$ region of $r v 0945$, respectively, suggesting probable additional deletion events in particular subbranches of these lineages.

In contrast, none of the almost 900 other genes specifically identified in the $M$. canettii genomes, and absent in the other MTBC genomes, were found in the L8 genome. The latter finding thus supports the close relationship with the previously known

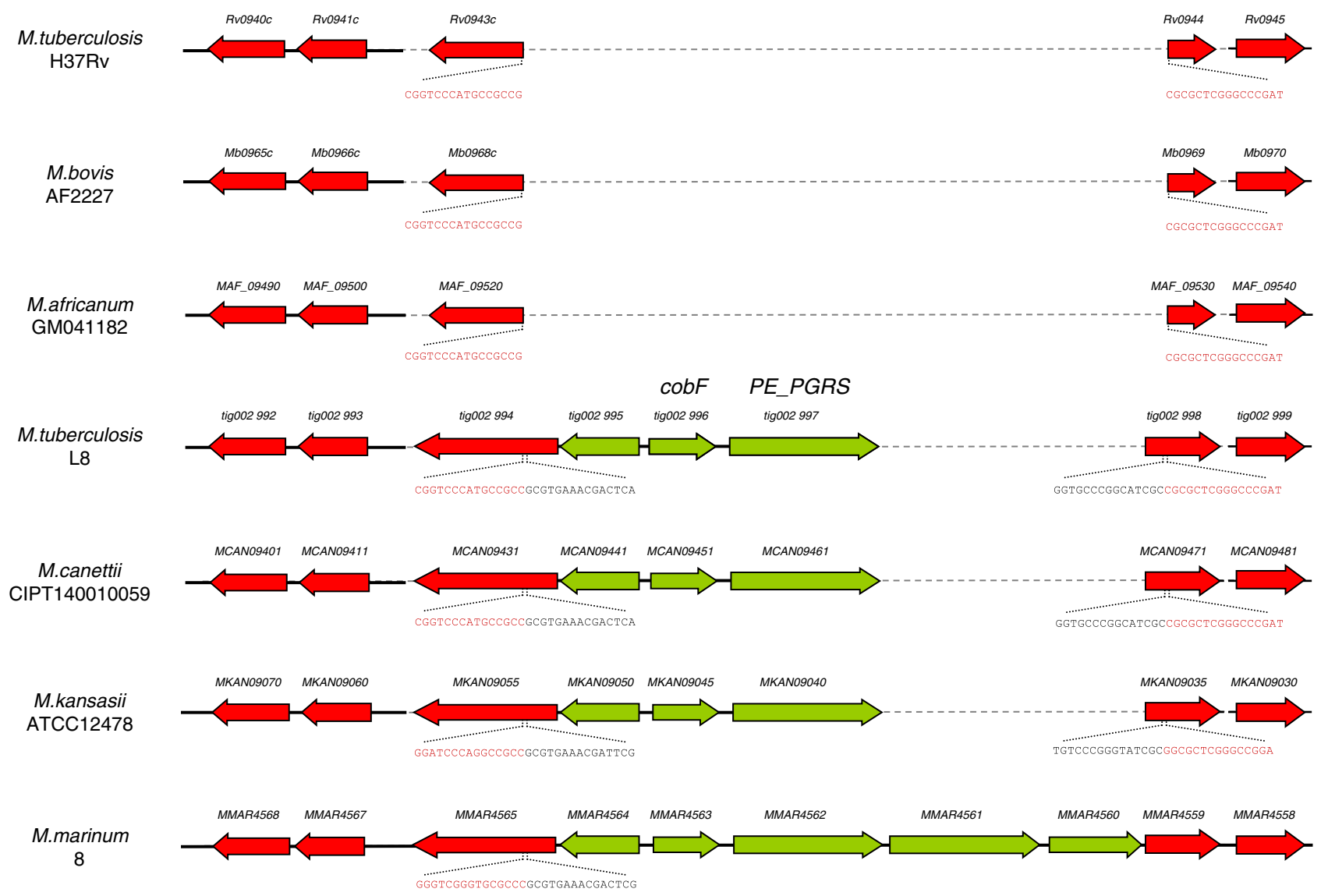

Fig. 4 Differential presence of the cobF region in specific Mycobacterium spp. genomes. Aligned genome segments showing the cobF gene region in M. tuberculosis L8, M. canettii CIPT140010059 (alias STB-A), M. kansasii ATCC12478 and M. marinum M strains, and the corresponding deletion in M. tuberculosis H37Rv, M. bovis AF2122/97 and M. africanum GM041182. Coding sequences of this region are shown in green, and flanking coding sequences in red. Sequences flanking the deletion point in truncated genes in $M$. tuberculosis, M. africanum and $M$. bovis, and in the cobF region present in $L 8, M$. canettii, M. kansasii and M. marinum are indicated in red and black, respectively. Dashed lines correspond to missing segment parts relatively to the longest segment found in $M$. marinum. 


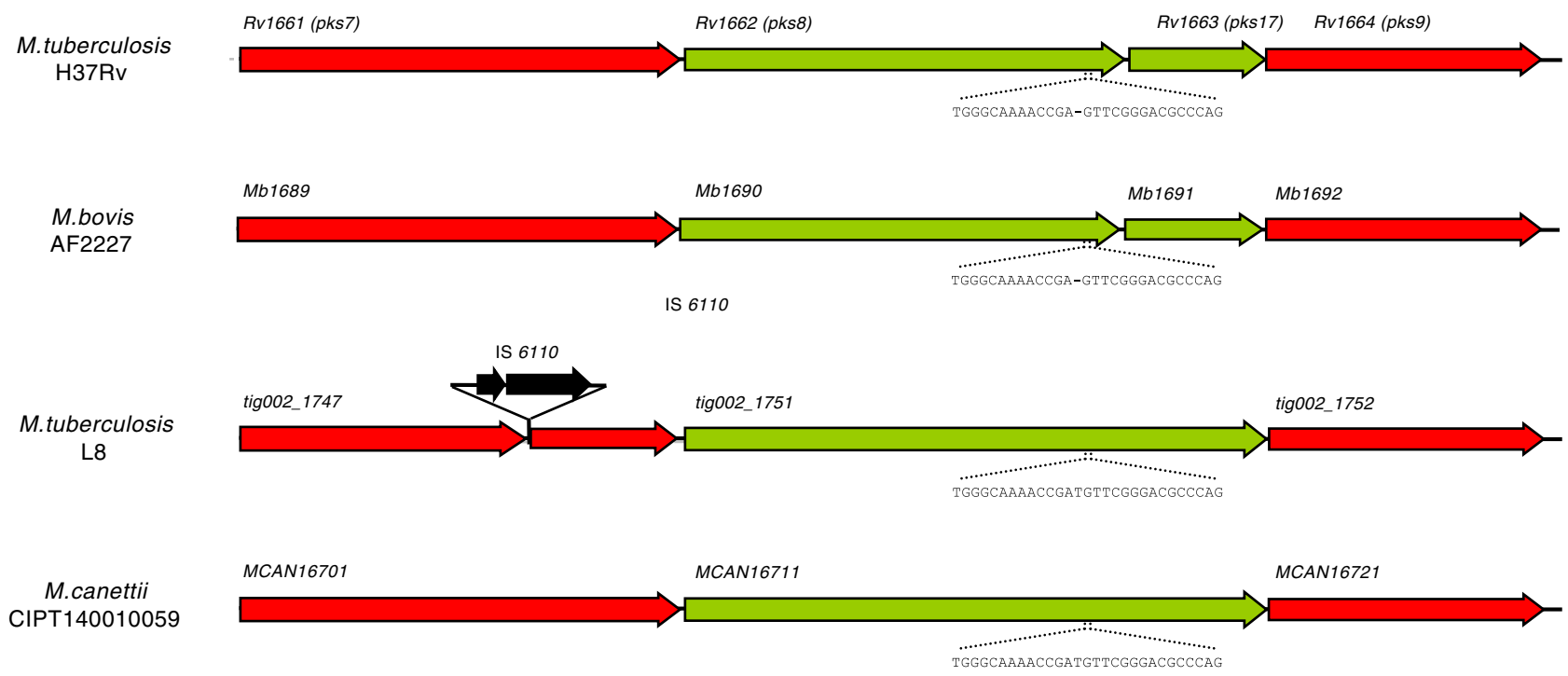

M.kansasii ATCC12478
MKAN_28240
MKAN_28245
MKAN_28250

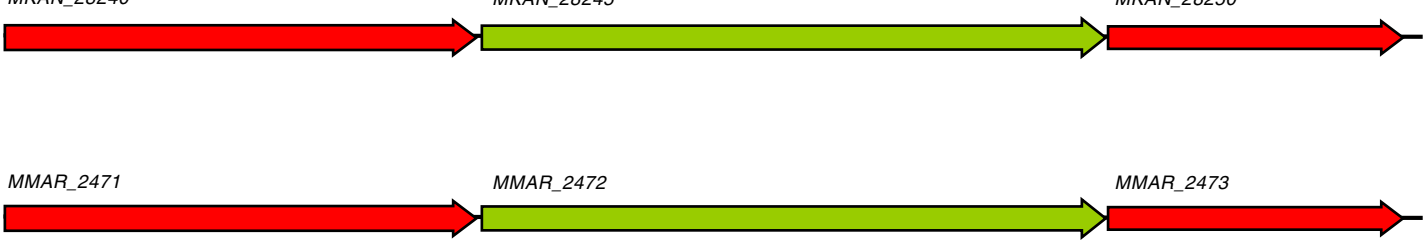

Fig. 5 Architecture of the pks8/17 region of specific Mycobacterium spp. genomes. Aligned genome segments showing the interrupted coding sequences pks8/17 in M. tuberculosis H37Rv and M. bovis AF2122/97, and complete pks8 genes in L8, M. canettii CIPT140010059 (alias STB-A) and M. kansasii ATCC12478. The coding sequences of pks8/17 and pks8 are shown in green, and flanking genes in red. Sequences flanking the 1-nucleotide deletion and resulting in a frameshift in $M$. tuberculosis complex strains are indicated.

MTBC lineages indicated by the SNP-based phylogeny, as well as the outgroup position of $M$. canettii relatively to the MTBC including L8.

Further evidence for the early branching of L8 relative to the rest of the MTBC comes from examination of interrupted coding sequences (ICDSs). These ICDSs correspond to frameshifts or inframe stop codons detected in genes that are intact in a common progenitor, thus putatively representing so-called molecular scars inherited during progressive pseudogenisation of the MTBC genomes $^{36,37}$. Four orthologues of MTBC ICDSs were previously found to be intact in the genomes of $M$. canettii strains, as well as in M. marinum and M. kansasii ${ }^{4}$. One of these four orthologues (pks8), which belongs to a multigene family encoding polyketide synthases involved in the biosynthesis of important cell envelope lipids $^{38}$, was also intact in the genomes of both L8 strains (Fig. 5 and Supplementary Data 3). Moreover, we found an additional orthologue of MTBC ICDSs (i.e. $r v 3899 c-r v 3900 c$ ), coding for a conserved hypothetical protein, which was intact in the genomes of M. canettii, M. kansasii, M. marinum and both L8 strains (Supplementary Data 3). These two molecular scars were also likely acquired by the other MTBC lineages after their divergence from the common progenitor shared with L8.

The assembled L8 genome also included 35 of the 50 genes (the exceptions are rv3513c encoding the probable fatty-Acid-Coa ligase FadD18 and 14 genes in the PhiRv1 region; see above) present in MTBC members but not found in any of the M. canettii genomes, including a number of genes putatively acquired through horizontal gene transfer by the common ancestor of the MTBC after its separation from M. canettii ${ }^{4}$ (Supplementary Data 4). This observation additionally supports both the close relationship with the previously known MTBC lineages and the outgroup position of $M$. canettii relatively to the MTBC including L8. Likewise, consistent with the rough colony morphotype of the Rwandan strain, both L8 strains displayed the single polyketidesynthase-encoding $p k s 5$ gene configuration shared by all MTBC members, instead of the dual pks5 conformation found in $M$. canettii strains involved in the smooth colony phenotype of the latter strains ${ }^{15}$. Thus, the recombination between the two pks5 genes and the loss of the intervening pap gene, thought to have resulted in surface remodelling and incremental gain of virulence after the phylogenetic separation from $M$. canettii ${ }^{15}$, already existed in the common progenitor of $\mathrm{L} 8$ and the rest of the MTBC. Moreover, both L8 strains also contained the intact TbD1 and RD9 regions, shared by the other 'ancestral' $M$. tuberculosis lineages (L1, L7) but subsequently lost by the so-called 'modern' lineages of M. tuberculosis (TbD1 lost in L2-4), M. africanum (L5 and L6) and the animal lineages (RD9) ${ }^{31}$.

In contrast to the highly clonal population structure of the MTBC, $M$. canettii strains are highly recombinogenic, as apparent from mosaic sequence arrangements in their genomes and functional DNA transfer between M. canettii strains mediated by a distributive conjugal transfer (DCT)-like mechanism ${ }^{4,39}$. However, no significant genome-wide recombination signal was detected by ClonalFrameML analysis ${ }^{40}$ between L8 and other MTBC strains (Supplementary Fig. 2). In particular, and in contrast to the numerous recombination segments in M. canettii ${ }^{4}$, the complete L8 genome only contained 26 possible recombination segments, yet the longest of these was $607 \mathrm{bp}$ and the average length was $142 \mathrm{bp}$.

\section{Discussion}

The discovery of L8 provides unique insights into an ancestor of the MTBC that existed after the pks5-recombination-mediated 
surface remodelling, which occurred after separation of the MTBC MRCA from the M. canettii clade, but preceded the loss of the $c o b F$ region and gene interruptions in a later common ancestor of the other MTBC lineages. The seeming restriction of this lineage to the African Great Lakes region represents new evidence supporting an origin for the MTBC in the eastern part of the African continent. These findings reinforce results from previous work suggesting an East-rather than a West African origin of the MTBC $3,4,9,11,41$.

A distinct ecological niche, linked to a potential environmental reservoir, has been hypothesised to explain the marked geographic restriction of $M$. canettii strains to the Horn of Africa, the lower persistence of these strains in infection models as well as their genome mosaicism implying multiple DNA recombination events within the $M$. canettii strain pool ${ }^{4,12}$. However, although our analysis is limited to two genomes identified to date, our results suggest that $\mathrm{L} 8$ is as clonal as the rest of the MTBC $^{3,14,34,42}$. Moreover, the observation that both L8 strains share two uncommon rifampicin- and isoniazid-resistance conferring mutations in $r p o B$ and inh $A$ suggests that multidrug resistance was already acquired in the common ancestor of these two strains. Isoniazid and rifampicin were introduced in TB treatments in the African Great Lakes region in the late fifties and 1983, respectively (Dr. Armand Van Deun, personal communication). These shared MDR-defining mutations, and the detection of these isolates in human patients in both cases (with reported absence of previous TB history for the Rwandan patient), suggest that these patients were infected with an alreadyresistant strain, which was exposed to drug selective pressure already decades ago and had been likely circulating in the community for some time. Overall, this pattern thus suggests humanto-human transmission rather than infection from a non-human source. Although based on only two initial strains, these results are consistent with the presumed scenario of a human rather than a zoonotic origin for the $\mathrm{MTBC}^{31,43}$.

Of note, given the above timeline of introduction of rifampicin and isoniazid in both countries, the 100 SNPs distance separating these two strains from their MRCA would imply a rapid molecular clock for L8, in the range of the upper bound of 2.2 SNPs/genome/year most recently estimated for other MTBC clades $^{44}$. However, this mutation rate cannot be confirmed until additional L8 samples are uncovered.

Remarkably, the absence of other L8 strains in datasets from Uganda, Rwanda and DRC, together comprising more than 2000 strains, suggests that L8 is rare even within the African Great Lakes region. Such scarcity is compatible with selective sweeps of later branching MTBC strains, introduced more recently into the region. Similar scenarios have also been proposed to explain the slow apparent replacement of MTBC L5 and L6 by L4 in West Africa ${ }^{45-47}$ and the restriction of L7 to Northern Ethiopia ${ }^{48}$.

Loss-of-function linked to the deletion of $c o b F$ is a plausible candidate molecular event involved in such a replacement scenario for L8. Indeed, loss-of-function appears to be an important mechanism driving the pathoadaptive evolution of the TB pathogen, as shown for the role of the loss of lipo-oligosaccharide production (via recombination in the pks5 locus) ${ }^{15}$ in the evolution towards increased virulence from $M$. canettii to MTBC strains. Likewise, loss of secretion of PPE-MPTR and PE_PGRS proteins by the type VII secretion system ESX-5 (via mutations of the ppe38 locus) has been involved in the hypervirulence of recent branches of L2 (alias 'modern' Beijing) strains ${ }^{49}$. The loss of the cobF region in the other MTBC lineages, inferred from comparative genomics with $M$. canettii and non-tuberculous mycobacteria ${ }^{4}$, was previously hypothesised to reflect enhanced adaptation to an intracellular parasitic lifestyle ${ }^{50}$. Indeed, the cobalamin/vitamin B12 synthesis pathway, of which the cobFencoded precorring-6a synthase is a component, represents a highly complex and energy consuming process with about 30 enzymatic steps ${ }^{51}$. Although the absence of this component may not entirely ablate cobalamin biosynthesis ${ }^{52,53}$, its loss might have resulted in gain of fitness and reflect enhanced pathogenic professionalisation, by economical reliance upon the mammalian host environment as source of vitamin B12. As an additional plausible but not necessarily mutually exclusive hypothesis, such selective sweep of L8 might have been (further) enhanced by the loss of theTbD1 region in later branching MTBC strains. This region, which we also found intact in L8, as is the case in the 'ancestral' $M$. tuberculosis lineages L1 and L7, encodes members of the mycobacterial membrane protein families MmpL. Very recent findings indicate that the loss of this region in later branching MTBC strains was also associated with a gain of virulence, and the deletion of TbD1 at the origin of the 'modern' M. tuberculosis lineages L2/L3/L4 has therefore been suggested as a key driver for their global epidemic spread ${ }^{54}$. If true, more recently emerged or introduced $c o b F$ - and TbD1-deleted strains might conceivably have largely outcompeted L8 strains. This hypothesis could be explored by assessing the growth and the virulence/fitness in cellular and animal models, of recombinant cobF- and/or TbD1-knock-out, as well as cobF- and/or TbD1knock-in strains, derived from the available Rwandan L8 strain and other MTBC strains, respectively.

In conclusion, our genomic data, on an as-yet-unknown ancestral stage between the MTBC and the putative progenitor pool of M. canettii-like mycobacteria, thus suggest further experiments to examine candidate molecular events potentially involved in the pathoadaptive evolution of $M$. tuberculosis. The discovery of such rare strains raises the possibility for the existence of further extant strains, especially in Eastern Africa, representing other clades further closing the biological gap between the MTBC and $M$. canettii.

\section{Methods}

Rwandan patient recruitment and ethics statement. The patient in Rwanda was recruited into, and gave informed consent for, the DIAMA study, which was approved by the Rwanda National Ethical committee (IRB 00001497 of IORG0001100; Ref No.0069/RNEC/2017). Consent to publish identifying information was obtained from the patient's representative.

Phenotypic characterisation. We studied conventional mycobacterial growth and biochemical characteristics including colony morphology, niacin production, nitrate reduction, $p$-nitro benzoic acid growth inhibition, catalase production, urea hydrolysis, Tween 80 hydrolysis and thiophene carboxylic acid hydrazide growth inhibition ${ }^{55}$. For comparative purpose, a reference set of the seven known humanadapted MTBC lineages ${ }^{56}$, together with $M$. canettii (BCCM/ITM500199), M. bovis (BCCM/ITM500324), M. bovis BCG (BCCM/ITM500101) and M. orygis (BCCM/ ITM501026) strains were processed with the novel strain isolated in Rwanda. Moreover, phenotypic drug-susceptibility testing to first- and second-line anti-TB drugs was done using the proportion method ${ }^{57}$. The strain isolated in Uganda was not available for phenotypic characterisation.

Targeted- and whole-genome sequencing of the Rwandan strain. Targeted sequencing was performed by using the Deeplex-MycTB assay ${ }^{58}$ (Genoscreen, France). Briefly, this assay relies on a 24-plexed PCR amplification of mycobacterial species identification ( $h s p 65$ ), genotyping (spoligotyping and phylogenetic single nucleotide polymorphisms (SNPs)) and $18 \mathrm{M}$. tuberculosis complex drug resistanceassociated gene targets. This test and short-read Illumina-based WGS were performed on the Rwandan strain as follows. A bead beating method was used to extract DNA from colonies as follows: a loopful colonies from LJ were suspended in TrisEDTA buffer, and heat inactivated at $95^{\circ} \mathrm{C}$ for $20 \mathrm{~min}$. After cooling at room temperature, the suspension was centrifuged at $20,000 \times \mathrm{g}$ for $30 \mathrm{~min}$ followed by discarding supernatant and $250 \mu \mathrm{l}$ of $10 \mathrm{mM}$ Tris- $\mathrm{HCl} \mathrm{pH} 7.8$ were added and briefly vortexed. Mixture was incubated at $95^{\circ} \mathrm{C}$ for $15 \mathrm{~min}$, then spun down briefly followed by transferring entire volume in a new microcentrifuge tube containing $0.5 \mathrm{~g}$ of zirconium beads (Sigma-Aldrich, St. Louis, USA). For destruction of the solid mycobacterial cell wall, the mixture was vortexed at high speed for at least $30 \mathrm{~s}$ followed by briefly spinning down and incubation at $-20^{\circ} \mathrm{C}$ for at least $30 \mathrm{~min}$. After 
thawing at room temperature, the mixture was briefly spun down and $200 \mu \mathrm{l}$ of supernatant was transferred to a new microcentrifuge tube. For gDNA concentration, $1 \mu \mathrm{l}$ of glycogen solution (Sigma-Aldrich, St. Louis, USA) was added followed by 0.1 volume of $3 \mathrm{M}$ sodium acetate at $\mathrm{pH} 5.2$ (Thermo Fisher Scientific, Waltham, MA USA), then 3 volume of $100 \%$ pre- cooled ethanol were added and vigorously vortexed for $10 \mathrm{~s}$. The mixture was incubated at $-20^{\circ} \mathrm{C}$ for $10 \mathrm{~min}$. After thawing at room temperature, the mixture was centrifuged at $15,000 \times g$ for $20 \mathrm{~min}$ followed by discarding supernatant, then $600 \mu \mathrm{l}$ of freshly prepared pre-cooled $70 \%$ ethanol was added followed by centrifugation at $15,000 \times g$ for $5 \mathrm{~min}$. Supernatant was discarded and the tube was air dried. gDNA was resuspended in $20 \mu \mathrm{l}$ of sterile molecular grade water. The yield was measured by Qubit dsDNA BR Assay Kit (Life Technologies, Carlsbad, USA).

Libraries of Deeplex-MycTB amplicons or genome fragments were constructed using the Nextera XT kit and sequenced on an Illumina MiSeq platform with paired end, 150-bp read lengths (Illumina, CA, USA). DNA extraction suitable for PacBio SMRT sequencing was performed using the Genomic DNA Buffer Set (Qiagen Inc, Germantown, Maryland, USA) as follows: colonies (70.3 $\pm 1.0 \mathrm{mg}$ ) from a one month old $\mathrm{LJ}$ were transferred into a $50 \mathrm{ml}$ falcon tube containing $3.5 \mathrm{ml}$ of the Qiagen buffer B1 and $70 \mu \mathrm{l}$ of $10 \mathrm{mg} / \mathrm{ml}$ RNAse (Life Technologies, Carlsbad, USA) solution followed by thoroughly vortex and heat inactivation of bacilli at $80^{\circ} \mathrm{C}$ for $1 \mathrm{~h}$. Vortexing at later stages was avoided to maintain high molecular weight DNA, by gently inverting or swirling instead. After cooling at room temperature, $100 \mu \mathrm{l}$ of $100 \mathrm{mg} / \mathrm{ml}$ lysozyme (Sigma-Aldrich, St. Louis, USA) was added and the tube was incubated at $37^{\circ} \mathrm{C}$ for $60 \mathrm{~min}$, followed by adding $1.2 \mathrm{ml}$ of $2.5 \mathrm{mg} / \mathrm{ml}$ of Proteinase K (MP Biomedicals, Santa Ana, USA) and again incubated at $37^{\circ} \mathrm{C}$ for $60 \mathrm{~min}$. For protein denaturation (nucleases and DNA- binding proteins), $1.2 \mathrm{ml}$ of buffer $\mathrm{B} 2$ was added to the mixture and placed overnight in horizontal shaker at $50{ }^{\circ} \mathrm{C}(30 \mathrm{RPM})$ alongside with Qiagen buffer QF. On the next day, a Qiagen Genomic-tip 100/G tip was placed over a $50 \mathrm{ml}$ falcon tube and equilibrated with $4 \mathrm{ml}$ of buffer QBT allowing the column to empty by gravity flow. The overnight incubated mixture was centrifuged at $3700 \times g, 4^{\circ} \mathrm{C}$ for $10 \mathrm{~min}$, followed by applying the supernatant to the equilibrated column allowing it to enter the resin by gravity flow. The column was washed by adding twice $7.5 \mathrm{ml}$ of buffer QC and then placed over a new clean $50 \mathrm{ml}$ falcon tube, and the DNA was eluted with $5 \mathrm{ml}$ pre-warmed QF buffer. For DNA precipitation, $3.5 \mathrm{ml}$ of isopropanol were added followed by centrifugation at $4500 \times g, 4^{\circ} \mathrm{C}$ for $20 \mathrm{~min}$ then the supernatant was gently discarded. gDNA was then washed with $1.5 \mathrm{ml}$ cold $\left(4^{\circ} \mathrm{C}\right) 70 \%$ ethanol, centrifuged at $10,000 \times g, 4^{\circ} \mathrm{C}$ for $15 \mathrm{~min}$ followed by discarding all supernatant and drying the pellet in the Speedvac for $5 \mathrm{~min}$ at the medium drying setting. Finally, $200 \mu \mathrm{l}$ of $10 \mathrm{mM}$ Tris-Cl, $\mathrm{pH} 8.0$ buffer was added and mixed gently by tapping and refrigeration overnight. On the next day, gDNA pellet was resuspended in the horizontal shaker at $65^{\circ} \mathrm{C}(250 \mathrm{RPM})$ for $15 \mathrm{~min}$. The purity and integrity of the gDNA were examined through $0.5 \%$ agarose gel electrophoresis and yield was measured using the NanoDrop 1000 Spectrophotometer (Thermo Fisher Scientific, Waltham, MA USA) and Qubit dsDNA BR Assay Kit (Life Technologies, Carlsbad, USA) according to manufacturer's instructions. Sequencing was performed on a PacBio RS II using the SMRT technology.

Deeplex-MycTB analysis and spoligotyping. Analysis of the Deeplex-MycTB sequencing data, including SNP calling and spoligotype identification, was performed by read mapping on $M$. tuberculosis $\mathrm{H} 37 \mathrm{Rv}$ sequence references, using a parameterised web application (GenoScreen $)^{58}$. Membrane-based spoligotyping was performed as described previously ${ }^{59}$.

Illumina whole-genome sequencing analysis. Raw genomic reads from the newly sequenced L8 genome from Rwanda and the L8 genome from Uganda (SAMN02567762) were processed as previously described ${ }^{60}$. Briefly, the reads were trimmed with Trimmomatic v0.33.2261 and reads larger than $20 \mathrm{bp}$ were kept. The software SeqPrep (https://github.com/jstjohn/SeqPrep) was used to identify and merge any overlapping paired-end reads. The resulting reads were aligned to the reconstructed ancestral sequence of the $\mathrm{MTBC}^{62}$ using the mem algorithm of BWA v0.7.1363. Duplicated reads were marked using the MarkDuplicates module of Picard v2.9.1 (https://github.com/broadinstitute/picard) and local realignment of reads around InDels was performed using the RealignerTargetCreator and IndelRealigner modules of GATK v3.4. $0^{64}$. SNPs were called with Samtools v1.2 mpileup ${ }^{65}$ and VarScan v2.4.1 ${ }^{66}$ using the following thresholds: minimum mapping quality of 20 , minimum base quality at a position of 20 , minimum read depth at a position of $7 \mathrm{X}$, maximum strand bias for a position $90 \%$.

The spoligotype pattern of the strain from Uganda was extracted in silico from the raw reads using $\mathrm{KvarQ}^{67}$.

Phylogenetic reconstruction. The maximum likelihood phylogeny was inferred with RAxML v.8.2.8 ${ }^{68}$ using an alignment containing only polymorphic sites and the branch lengths of the tree were rescaled using invariant sites ${ }^{44,69}$.

A position was considered polymorphic if at least one genome had a SNP at that position. Deletions and positions not called according to the minimum threshold of $7 \times$ were encoded as gaps. We excluded positions with more than $20 \%$ missing data, positions falling in PE-PGRS genes, phages, insertion sequences and in regions with at least $50 \mathrm{bp}$ identity to other regions in the genome. We also excluded variable positions falling in drug resistance-related genes. The phylogeny was computed using the general time-reversible model of sequence evolution (-m GTRCAT -V options), 100 bootstrap inferences and $M$. canettii (SRR011186) were used as an outgroup to root the phylogeny.

\section{Whole-genome de novo assembly, annotation and comparative genomics.}

Raw PacBio reads obtained from the Rwandan strain were assembled with Canu $\mathrm{v} 1.6^{70}$, using default settings and an expected genome size of $4.4 \mathrm{Mbp}$, typical of MTBC strains. After discarding 60,272 reads below minimal quality parameters, 106,681 reads were used for the assembly. On the basis of the expected genome size, the average coverage depth was estimated at $186 \times$ using raw reads, and $39 \times$ and $38 \times$ using corrected and trimmed reads, respectively. The obtained unique contig of 4,387,285 bp was circularised with Circlator v1.5.5 $5^{71}$ using default settings, resulting in an assembly of 4,379,493 bp. Additional sequence verification and correction was then performed by mapping Illumina reads obtained from the same strain, using pacbio-utils v0.2 $2^{72}$ (https://github.com/douglasgscofield/PacBioutilities) and snippy v4.4 $4^{73}$ (https://github.com/tseemann/snippy). Alignments of the final assembly were performed against an ensemble of complete genome sequences available from 38 strains of tubercle bacilli. This set included $34 \mathrm{M}$. tuberculosis strains from lineages 1, 2, 3 and 4 (comprising H37Rv), M. africanum L6 GM041182, M. bovis AF2122/97, as well as the closest STB-A (CIPT140010059) and most distant (STB-K) M. canettii strains (Supplementary Data 5). Comparative alignments and genome annotation were performed based on BLAST searches and analysis of gene synteny, using Artemis and Artemis comparison tool ${ }^{74}$, as well as a custom Multiple Annotation of Genomes and Differential Analysis (MAGDA) software previously used for annotation of $M$. canettii and Helicobacter pylori genomes ${ }^{4,75}$. Comparisons with orthologues from $M$. canettii STB-D, -E, -G, -H, -I and $-\mathrm{J}$ in addition to STB-A and $-\mathrm{K}$, and from M. marinum type strain $\mathrm{M}$ and $M$. kansasii genomes were additionally done using the Microscope platform v3.13.376 When applicable, annotations were transferred from those of M. tuberculosis or $M$. canettii orthologs in the TubercuList/Mycobrowser database, using BLAST matches of $>90 \%$ protein sequence identity, an alignable region of $>80 \%$ of the shortest protein length in pairwise comparisons and visual inspection of the gene synteny. Genome completeness was assessed using $\mathrm{CheckM}^{77}$ using the lineage-specific workflow and Mycobacterium as the genus. The PacBio assembled Rwandan strain was found to have $98.74 \%$ completeness and $0 \%$ contamination or strain heterogeneity, making it suitable for further analyses.

ACT comparison files were generated using MAUVE 2015-02-25 software to visualise the genome-wide distribution of SNP densities between the assembled L8 genome from Rwanda and M. tuberculosis $\mathrm{H} 37 \mathrm{Rv}$ and $M$. canettii STB-A and STB-K genomes. Recombination between L8 and other MTBC lineages or M. canettii was assessed from a progressive MAUVE alignment of the PacBio assembled L8 genome and previously published closed genomes ${ }^{73}$ using ClonalFrameML ${ }^{40}$. To further assess the phylogenetic placement of the Lineage 8 strain, a core gene alignment was constructed using the completed genomes of the Rwandan L8 strain, representatives of the MTBC lineages 1-4 and 6, $M$. canettii, M. bovis, M. marinum (accession GCF_000419315.1) and M. kansassii (accession GCF_000157895.3). The GFF files of each genome were input to roary ${ }^{78}$ with an $80 \%$ identity cut-off, as has been done for previous genus-level mycobacterial core alignments ${ }^{79}$. A phylogenetic tree was constructed from the core gene superalignment using RAxML-NG v0.9.0 ${ }^{80}$ under the GTR + Gamma model of evolution with 20 starting trees. Bootstrapping was run until autoMRE converged with a value of 0.03 (50 replicates).

Reporting summary. Further information on research design is available in the Nature Research Reporting Summary linked to this article.

\section{Data availability}

The complete genome sequence of the L8 strain from Rwanda was deposited in the NCBI repository under project PRJNA598991 with SRR10828835 and SRR10828834 accession codes for Illumina- and PacBio-derived genome sequences, respectively. The strain can be requested from the BCCM/ITM [http://bccm.belspo.be/] collection with accession code ITM-500961.

Received: 21 January 2020; Accepted: 13 May 2020; Published online: 09 June 2020

\section{References}

1. Gagneux, S. Ecology and evolution of Mycobacterium tuberculosis. Nat. Rev. Microbiol. 16, 202-213 (2018).

2. World Health Organization. Global Tuberculosis Report 2018 (WHO, 2018).

3. Comas, I. et al. Out-of-Africa migration and Neolithic coexpansion of Mycobacterium tuberculosis with modern humans. Nat. Genet. 45, 1176-1182 (2013). 
4. Supply, P. et al. Genomic analysis of smooth tubercle bacilli provides insights into ancestry and pathoadaptation of Mycobacterium tuberculosis. Nat. Genet. 45, 172-179 (2013).

5. Bos, K. I. et al. Pre-Columbian mycobacterial genomes reveal seals as a source of New World human tuberculosis. Nature 514, 494-497 (2014).

6. Brites, D. et al. A new phylogenetic framework for the animal-adapted Mycobacterium tuberculosis complex. Front. Microbiol. 9, 2820 (2018).

7. Rutaihwa, L. K. et al. Multiple introductions of Mycobacterium tuberculosis lineage 2-Beijing into Africa over centuries. Front. Ecol. Evolution 7, 112 (2019).

8. O'Neill, M. B. et al. Lineage specific histories of Mycobacterium tuberculosis dispersal in Africa and Eurasia. Mol. Ecol. 28, 3241-3256 (2019).

9. Wirth, T. et al. Origin, spread and demography of the Mycobacterium tuberculosis complex. PLoS Pathog. 4, e1000160 (2008).

10. Gagneux, S. et al. Variable host-pathogen compatibility in Mycobacterium tuberculosis. Proc. Natl Acad. Sci. USA 103, 2869-2873 (2006).

11. Gutierrez, M. C. et al. Ancient origin and gene mosaicism of the progenitor of Mycobacterium tuberculosis. PLoS Pathog. 1, e5 (2005)

12. Blouin, Y. et al. Progenitor "Mycobacterium canettii" clone responsible for lymph node tuberculosis epidemic, Djibouti. Emerg. Infect. Dis. 20, 21-28 (2014).

13. Coscolla, M. \& Gagneux, S. Consequences of genomic diversity in Mycobacterium tuberculosis. Semin. Immunol. 26, 431-444 (2014).

14. Boritsch, E. C. et al. Key experimental evidence of chromosomal DNA transfer among selected tuberculosis-causing mycobacteria. Proc. Natl Acad. Sci. USA 113, 9876-9881 (2016).

15. Boritsch, E. C. et al. pks5-recombination-mediated surface remodelling in Mycobacterium tuberculosis emergence. Nat. Microbiol. 1, 15019 (2016).

16. Mortimer, T. D. \& Pepperell, C. S. Genomic signatures of distributive conjugal transfer among mycobacteria. Genome Biol. Evol. 6, 2489-2500 (2014).

17. Helb, D. et al. Rapid detection of Mycobacterium tuberculosis and rifampin resistance by use of on-demand, near-patient technology. J. Clin. Microbiol. 48, 229-237 (2010).

18. Ng, K. C. S. et al. Automated algorithm for early identification of rifampicinresistant tuberculosis transmission hotspots in Rwanda [abstract]. Int. J. Tuberc. Lung Dis. 22, 605 (2018).

19. Torrea, G. et al. Variable ability of rapid tests to detect Mycobacterium tuberculosis rpoB mutations conferring phenotypically occult rifampicin resistance. Sci. Rep. 9, 11826 (2019).

20. Trébucq, A. et al. Treatment outcome with a short multidrug-resistant tuberculosis regimen in nine African countries. Int. J. Tuberc. Lung Dis. 22, $17-25$ (2018)

21. Coll, F. et al. A robust SNP barcode for typing Mycobacterium tuberculosis complex strains. Nat. Commun. 5, 4812 (2014).

22. Couvin, D., David, A., Zozio, T. \& Rastogi, N. Macro-geographical specificities of the prevailing tuberculosis epidemic as seen through SITVIT2, an updated version of the Mycobacterium tuberculosis genotyping database. Infect. Genet. Evol. https://doi.org/10.1016/j.meegid.2018.12.030 (2018).

23. Wanzala, S. I. et al. Retrospective analysis of archived pyrazinamide resistant Mycobacterium tuberculosis complex isolates from Uganda-evidence of interspecies transmission. Microorganisms 7, 221 (2019).

24. Stinear, T. P. et al. Insights from the complete genome sequence of Mycobacterium marinum on the evolution of Mycobacterium tuberculosis. Genome Res. 18, 729-741 (2008).

25. Wang, J. et al. Insights on the emergence of Mycobacterium tuberculosis from the analysis of Mycobacterium kansasii. Genome Biol. Evol. 7, 856-870 (2015).

26. Ssengooba, W. et al. Whole genome sequencing to complement tuberculosis drug resistance surveys in Uganda. Infect. Genet. Evol. 40, 8-16 (2016).

27. Carpels, G. et al. Drug resistant tuberculosis in sub-Saharan Africa: an estimation of incidence and cost for the year 2000. Tuber. Lung Dis. 76, 480-486 (1995).

28. Umubyeyi, A. N. et al. Results of a national survey on drug resistance among pulmonary tuberculosis patients in Rwanda. Int. J. Tuberc. Lung Dis. 11, 189-194 (2007).

29. World Health Organization. WHO | Global tuberculosis report 2016 (WHO, 2019).

30. Yang, T. et al. Pan-genomic study of Mycobacterium tuberculosis reflecting the primary/secondary genes, generality/individuality, and the interconversion through copy number variations. Front. Microbiol. 9, 1886 (2018).

31. Brosch, R. et al. A new evolutionary scenario for the Mycobacterium tuberculosis complex. Proc. Natl Acad. Sci. USA 99, 3684-3689 (2002).

32. Tsolaki, A. G. et al. Functional and evolutionary genomics of Mycobacterium tuberculosis: Insights from genomic deletions in 100 strains. Proc. Natl Acad. Sci. USA 101, 4865-4870 (2004).

33. Brosch, R. et al. Genomic analysis reveals variation between Mycobacterium tuberculosis $\mathrm{H} 37 \mathrm{Rv}$ and the attenuated M. tuberculosis H37Ra strain. Infect. Immun. 67, 5768-5774 (1999).
34. Hirsh, A. E., Tsolaki, A. G., DeRiemer, K., Feldman, M. W. \& Small, P. M. Stable association between strains of Mycobacterium tuberculosis and their human host populations. Proc. Natl Acad. Sci. USA 101, 4871-4876 (2004).

35. Chiner-Oms, Á. Genomic determinants of speciation and spread of the Mycobacterium tuberculosis complex. Sci. Adv. 5, eaaw3307 (2019).

36. Deshayes, C. et al. Detecting the molecular scars of evolution in the Mycobacterium tuberculosis complex by analyzing interrupted coding sequences. BMC Evol. Biol. 8, 78 (2008).

37. Smith, N. H., Hewinson, R. G., Kremer, K., Brosch, R. \& Gordon, S. V. Myth and misconceptions: the origin and evolution of Mycobacterium tuberculosis. Nat. Rev. Microbiol. 7, 537-544 (2009).

38. Etienne, G. et al. Identification of the polyketide synthase involved in the biosynthesis of the surface-exposed lipooligosaccharides in mycobacteria. $J$. Bacteriol. 191, 2613-2621 (2009).

39. Boritsch, E. C. \& Brosch, R. in Tuberculosis and the Tubercle Bacillus 2nd edn, Vol. 4 495-515 (American Society of Microbiology, 2016).

40. Didelot, X. \& Wilson, D. J. ClonalFrameML: efficient inference of recombination in whole bacterial genomes. PLoS Comput. Biol. 11, e1004041 (2015).

41. Hershberg, R. et al. High functional diversity in Mycobacterium tuberculosis driven by genetic drift and human demography. PLoS Biol. 6, e311 (2008).

42. Supply, P. et al. Linkage disequilibrium between minisatellite loci supports clonal evolution of Mycobacterium tuberculosis in a high tuberculosis incidence area. Mol. Microbiol. 47, 529-538 (2003).

43. Mostowy, S., Cousins, D., Brinkman, J., Aranaz, A. \& Behr, M. A. Genomic deletions suggest a phylogeny for the Mycobacterium tuberculosis complex. J. Infect. Dis. 186, 74-80 (2002).

44. Menardo, F., Duchêne, S., Brites, D. \& Gagneux, S. The molecular clock of Mycobacterium tuberculosis. PLoS Pathog. 15, e1008067 (2019).

45. Niobe-Eyangoh, S. N. et al. Genetic biodiversity of Mycobacterium tuberculosis complex strains from patients with pulmonary tuberculosis in Cameroon. J. Clin. Microbiol. 41, 2547-2553 (2003).

46. Godreuil, S. et al. First molecular epidemiology study of Mycobacterium tuberculosis in Burkina Faso. J. Clin. Microbiol. 45, 921-927 (2007).

47. Groenheit, R. et al. The Guinea-Bissau family of Mycobacterium tuberculosis complex revisited. PLoS ONE 6, e18601 (2011).

48. Comas, I. et al. Population genomics of Mycobacterium tuberculosis in Ethiopia contradicts the virgin soil hypothesis for human tuberculosis in SubSaharan Africa. Curr. Biol. 25, 3260-3266 (2015).

49. Ates, L. S. et al. Mutations in ppe38 block PE_PGRS secretion and increase virulence of Mycobacterium tuberculosis. Nat. Microbiol. 3, 181-188 (2018).

50. Boritsch, E. C. et al. A glimpse into the past and predictions for the future: the molecular evolution of the tuberculosis agent. Mol. Microbiol. 93, 835-852 (2014).

51. Martens, J. H., Barg, H., Warren, M. \& Jahn, D. Microbial production of vitamin B12. Appl. Microbiol. Biotechnol. 58, 275-B285 (2002).

52. Gopinath, $\mathrm{K}$. et al. A vitamin $\mathrm{B}_{12}$ transporter in Mycobacterium tuberculosis. Open Biol. 3, 120175 (2013).

53. Minias, A., Minias, P., Czubat, B. \& Dziadek, J. Purifying selective pressure suggests the functionality of a vitamin B12 biosynthesis pathway in a global population of Mycobacterium tuberculosis. Genome Biol. Evol. 10, 2326-2337 (2018).

54. Bottai, D. et al. TbD1 deletion as a driver of the evolutionary success of modern epidemic Mycobacterium tuberculosis lineages. Nat. Commun. 11, $1-14(2020)$

55. LEÃO, S. C. et al. Practical Handbook for the Phenotypic and Genotypic Identification of Mycobacteria, https://www.scienceopen.com/document? vid $=$ f4807bef-1c8d-431e-be6a-bced8cb452b9 (Vanden Broele, Brugge, Belgium, 2004).

56. Borrell, S. et al. Reference set of Mycobacterium tuberculosis clinical strains: a tool for research and product development. PLOS ONE 14, e0214088 (2019).

57. Kent, P. \& Kubica, G. Public Health Mycobacteriology: A Guide for the Level III Laboratory (US Department of Health and Human Services, Centres for Disease Control, 1985).

58. Makhado, N. A. et al. Outbreak of multidrug-resistant tuberculosis in South Africa undetected by WHO-endorsed commercial tests: an observational study. Lancet Infect. Dis. 18, 1350-1359 (2018).

59. van der Zanden, A. G. M. et al. Improvement of differentiation and interpretability of spoligotyping for Mycobacterium tuberculosis complex isolates by introduction of new spacer oligonucleotides. J. Clin. Microbiol. 40, 4628-4639 (2002)

60. Menardo, F. et al. Treemmer: a tool to reduce large phylogenetic datasets with minimal loss of diversity. BMC Bioinformatics 19, 164 (2018).

61. Bolger, A. M., Lohse, M. \& Usadel, B. Trimmomatic: a flexible trimmer for Illumina sequence data. Bioinformatics 30, 2114-2120 (2014).

62. Comas, I. et al. Human T cell epitopes of Mycobacterium tuberculosis are evolutionarily hyperconserved. Nat. Genet. 42, 498-503 (2010). 
63. Li, H. \& Durbin, R. Fast and accurate short read alignment with BurrowsWheeler transform. Bioinformatics 25, 1754-1760 (2009).

64. McKenna, A. et al. The Genome Analysis Toolkit: a MapReduce framework for analyzing next-generation DNA sequencing data. Genome Res. 20 1297-1303 (2010).

65. Li, H. A statistical framework for SNP calling, mutation discovery, association mapping and population genetical parameter estimation from sequencing data. Bioinformatics 27, 2987-2993 (2011).

66. Koboldt, D. C. et al. VarScan 2: somatic mutation and copy number alteration discovery in cancer by exome sequencing. Genome Res. 22, 568-576 (2012).

67. Steiner, A., Stucki, D., Coscolla, M., Borrell, S. \& Gagneux, S. KvarQ: targeted and direct variant calling from fastq reads of bacterial genomes. BMC Genomics 15, 881 (2014)

68. Stamatakis, A. RAxML version 8: a tool for phylogenetic analysis and postanalysis of large phylogenies. Bioinformatics 30, 1312-1313 (2014).

69. Duchene, S. et al. Inferring demographic parameters in bacterial genomic data using Bayesian and hybrid phylogenetic methods. BMC Evolut. Biol. 18, 95 (2018).

70. Koren, S. et al. Canu: scalable and accurate long-read assembly via adaptive kmer weighting and repeat separation. Genome Res. 27, 722-736 (2017).

71. Hunt, M. et al. Circlator: automated circularization of genome assemblies using long sequencing reads. Genome Biol. 16, 294 (2015).

72. Scofield, D. G. GitHub - douglasgscofield/PacBio-utilities: collection of utilities for working with PacBio-based assemblies. https://github.com/douglasgscofield/ PacBio-utilities (2015).

73. Seemann, T. GitHub - tseemann-snippy Rapid bacterial SNP calling and core genome alignments. https://github.com/tseemann/snippy (2015).

74. Carver, T. et al. Artemis and ACT: viewing, annotating and comparing sequences stored in a relational database. Bioinformatics 24, 2672-2676 (2008).

75. Li, H. et al. East-Asian Helicobacter pylori strains synthesize heptan-deficient lipopolysaccharide. PLoS Genet. 15, e1008497 (2019).

76. Médigue, C. et al. MicroScope-an integrated resource for community expertise of gene functions and comparative analysis of microbial genomic and metabolic data. Brief. Bioinformatics 20, 1071-1084 (2019).

77. Parks, D. H., Imelfort, M., Skennerton, C. T., Hugenholtz, P. \& Tyson, G. W. CheckM: assessing the quality of microbial genomes recovered from isolates, single cells, and metagenomes. Genome Res. 25, 1043-1055 (2015).

78. Page, A. J. et al. Roary: rapid large-scale prokaryote pan genome analysis. Bioinformatics 31, 3691-3693 (2015).

79. Fedrizzi, T. et al. Genomic characterization of Nontuberculous Mycobacteria. Sci. Rep. 7, 45258 (2017)

80. Kozlov, A. M., Darriba, D., Flouri, T., Morel, B. \& Stamatakis, A. RAxML-NG: a fast, scalable and user-friendly tool for maximum likelihood phylogenetic inference. Bioinformatics 35, 4453-4455 (2019).

\section{Acknowledgements}

Calculations were partially performed at sciCORE (http://scicore.unibas.ch/) scientific computing core facility at University of Basel. This work was supported by EDCTP2 grant DRIA2014-326-DIAMA of the European Union, the Belgian General Directorate for Development Cooperation (PhD fellowship to J.C.S.N.), Grant ANR-16-CE35-0009 from Agence Nationale de la Recherche, the Swiss National Science Foundation (Grants
310030_188888, IZRJZ3_164171, IZLSZ3_170834 and CRSII5_177163), and the European Research Council (309540-EVODRTB). The views and opinions of authors expressed herein do not necessarily state or reflect those of EDCTP. The funders had no role in study design, data collection and analysis, decision to publish or preparation of the manuscript.

\section{Author contributions}

S.G., P.S., C.M., B.C.d.J., L.R. and J.C.S.N. designed the study. P.S., J.C.S.N., C.L., M.M., C.M. and S.G. analysed data and wrote the manuscript, with comments from all authors A.J. and C.M. performed the assembly of sequences. M.M. annotated the L8 genome C.L., F.M., D.B. and A.J. performed SNP analyses and phylogenetic reconstruction. M.M., R.A. and P.S. conducted comparative analyses of complete mycobacterial genomes, with support from C.L. and O.T. J.C.S.N., E.B.N., Y.M.H., J.B.M., W.M., K.F. and M.D. performed and/or analysed data from mycobacterial isolation, growth assays, phenotypic characterisation and/or molecular tests. S.D., C.G., J.C.S.N., E.B.N., E.A., W.S. and M.K.K. conducted targeted deep sequencing analyses and/or provided Xpert data. B.C.d.J and D.A. run the DIAMA trial.

\section{Competing interests}

P.S. was a consultant of Genoscreen; C.G. and S.D. were employees of the same company. The other authors declare no competing interests.

\section{Additional information}

Supplementary information is available for this paper at https://doi.org/10.1038/s41467 020-16626-6.

Correspondence and requests for materials should be addressed to S.G., C.J.M. or P.S.

Peer review information Nature Communications thanks Prabhu Patil and the other, anonymous, reviewers for their contribution to the peer review of this work. Peer reviewer reports are available.

Reprints and permission information is available at http://www.nature.com/reprints

Publisher's note Springer Nature remains neutral with regard to jurisdictional claims in published maps and institutional affiliations.

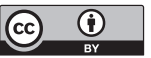

Open Access This article is licensed under a Creative Commons Attribution 4.0 International License, which permits use, sharing, adaptation, distribution and reproduction in any medium or format, as long as you give appropriate credit to the original author(s) and the source, provide a link to the Creative Commons license, and indicate if changes were made. The images or other third party material in this article are included in the article's Creative Commons license, unless indicated otherwise in a credit line to the material. If material is not included in the article's Creative Commons license and your intended use is not permitted by statutory regulation or exceeds the permitted use, you will need to obtain permission directly from the copyright holder. To view a copy of this license, visit http://creativecommons.org/ licenses/by/4.0/.

(C) The Author(s) 2020 